\title{
Pro-IL-18 secreted by keratinocytes detects the group A
}

\section{streptococcal protease SpeB}

5 Anders F. Johnson ${ }^{1 *}$, Jenna S. Sands ${ }^{1 *}$, Keya Trivedi ${ }^{1}$, Raedeen Russell ${ }^{1}$, Doris L. LaRock ${ }^{1}$, Christopher N.

6 LaRock $^{1 \#}$

7

$8{ }^{1}$ Department of Microbiology and Immunology and Department of Medicine, Emory School of Medicine,

9 Atlanta, GA, United States of America

10

11 Running head: Group A Streptococcus activation of IL-18

12

13 \#Corresponding author:

14 Christopher LaRock, Emory University School of Medicine, 1510 Clifton Road NE, Suite 3019, Atlanta,

15 GA 30322.+1-404-727-1574. christopher.larock@emory.edu

16

17

18 *Equal contributions

19 


\section{Abstract}

21 Group A Streptococcus (GAS, Streptococcus pyogenes) is a professional human pathogen that commonly

22 infects the skin. Keratinocytes are one of the first cells to contact GAS, and by inducing inflammation, they

23 can initiate the earliest immune responses to pathogen invasion. Here, we characterized the

24 proinflammatory cytokine repertoire produced by primary human keratinocytes and surrogate cell lines

25 commonly used in vitro. Infection induces several cytokines and chemokines, but keratinocytes

26 constitutively secrete IL-18 in a form that is inert (pro-IL-18) and lacks proinflammatory activity.

27 Canonically, IL-18 activation and secretion are coupled through a single proteolytic event that is regulated

28 intracellularly by the inflammasome protease caspase-1 in myeloid cells. The pool of extracellular pro-IL-

2918 generated by keratinocytes is poised to sense extracellular proteases and is directly processed into a

30 mature active form by $\mathrm{SpeB}$, a secreted GAS protease that is a critical virulent factor during skin infection.

31 This mechanism contributes to the proinflammatory response against GAS, resulting in $\mathrm{T}$ cell activation

32 and the secretion of IFN- $\gamma$ that restrict GAS growth. Other major bacterial pathogens and microbiota of this

33 skin did not have significant IL-18-maturing ability. Taken together, these results suggest keratinocyte-

34 secreted IL-18 is a sentinel that sounds an early alarm that is highly sensitive to GAS, yet tolerant to noninvasive members of the microbiota. 
37 The skin provides the first resistance to infectious, chemical, and physical insults. The obligate human pathogen Group A Streptococcus (GAS, Streptococcus pyogenes) colonizes oropharyngeal mucosa and epidermal surfaces, specifically adhere to and invading skin epithelial cells and keratinocytes [1-4]. Beyond superficial infections like impetigo, further tissue invasion can lead to cellulitis, sepsis, and necrotizing fasciitis. Invasive infections, and immune-mediated sequalae like rheumatic heart disease, are responsible

for an estimated half a million annual deaths globally [5]. These infections are highly inflammatory, which contributes to their pathology and complicates their treatment, and several lines of evidence suggest GAS use this inflammation to promote replication and transmission [6-9]

Interleukin-18 (IL-18) is a proinflammatory cytokine that induces cell-mediated immunity [10]. IL-18 is detected by the IL-18R/IL-18RAP (IL18R1/IL1R7, IL-18R $\alpha / \mathrm{IL}-18 \mathrm{R} \beta$ ) receptor complex and works with IL-12 to induce IFN- $\gamma$ and Th1-type responses from T cells, NK cells, and dendritic cells [11-13]. Accordingly, IL-18 is important in the host defense against Salmonella Typhimurium [14], Shigella flexneri [15], Yersinia enterocolitica [16], Listeria monocytogenes [17], Burkholderia pseudomallei [18], Mycobacterium tuberculosis [19], Streptococcus pneumoniae [20], and group B Streptococcus [21]. Newlysynthesized IL-18 (pro-IL-18) is inert and requires removal of an amino-terminal autoinhibitory domain, canonically by the host protease caspase- $1[11,12]$. Caspase- 1 is itself regulated by the inflammasome complex, which in myeloid cells can also regulate IL-1 $\beta$ maturation and the cell death program pyroptosis [22]. Caspase-8 [23], granzyme B [24], chymase [25], proteinase 3 [26], and neutrophil elastase [27] also cleave pro-IL-18, though their physiologic relevance in IL-18 activation remains to be established. Human keratinocytes constitutively express and release pro-IL-18 [28-31], suggesting the skin as an anatomical location where these or other extracellular proteases may participate in IL-18 activation.

We hypothesized that GAS interactions with human keratinocytes contributes to the proinflammatory storm observed during infection. This study shows that keratinocytes release numerous proinflammatory cytokines during GAS infection, including pro-IL-18, which is directly matured by the SpeB cysteine 
protease of GAS. This mechanism supports a model wherein SpeB acting as a "bacterial caspase" that proteolytically activates proinflammatory cytokines of the IL-1 family, which in term may limit GAS invasive infection.

\section{Results}

\section{GAS induces keratinocytes secretion of proinflammatory cytokines}

The epithelium is one of the first body tissues GAS will contact. Therefore, the resident cells provide one of the earliest responses to infection by producing antimicrobial effectors and proinflammatory cytokines and chemokines that coordinate immune response [32-35]. To assess the cytokine repertoire elicited by GAS, we infected cultured and primary cells epithelial cells with M1T1 GAS 5448, a highly virulent strain associated with modern epidemic invasive infections [36]. Similar to previous observations [32,33,37], we observed robust secretion of MIF, IL-8, and other proinflammatory cytokines and chemokines by infected HaCaT keratinocytes (Figure 1a). Detroit 562 human pharyngeal epithelial cells, HEp-2 human laryngeal epithelial cells, and A-431 human keratinocytes all secreted a similar, nonredundant, cytokine profile. However, compared to each of these cell lines, human primary keratinocytes expressed a greater diversity in CXC-family chemokines that were absent with any individual cell line and the pro-inflammatory cytokine IL-1 $\alpha$ (Figure 1a). In contrast, human primary endothelial cells also secreted cytokines and chemokines but had a distinct repertoire. No cell produced detectable CCL1, CCL5, G-CSF, IFN- $\gamma$, IL-1 $\beta$, IL-2, IL-4, IL-5, IL-10, IL-12, IL-13, IL-16, IL-17A, IL-17E, IL-21, IL-27, IL-32 $\alpha$, MIP-1,TNF- $\alpha$. Primary keratinocytes and endothelial cells each formed a distinct group relative to all cell lines by multivariate analysis (Figure 1b), due to each secreting a distinct cytokine profile (Figure 1c). 
One cytokine of particular interest to us was IL-18, an IL-1 family cytokine that bridges innate and adaptive immune responses stimulate IFN- $\gamma$ production by T and NK cells [10]. In immune cells, IL-18 release is conventionally regulated by the inflammasome protease caspase-1 alongside the cell death program, pyroptosis [22]. In contrast, healthy keratinocytes have been seen to constitutively release IL-18 [29], consistent with our observation that GAS infection was not required for IL-18 secretion (Figure 2a). Since chronic IL-18 signaling would be pathological, we next examined whether the activity of IL-18 secreted by keratinocytes. The determinant of activity for cytokines that are post-translationally regulated by proteolysis is not necessarily specific to a single regulator, and is exclusively whether the product formed is able to bind its cognate receptor and stimulate receptor signaling [6]. To measure whether keratinocyte-released IL-18 had biological activity, we used HEK-Blue ${ }^{\mathrm{TM}}$ IL-18 Reporter Cells, which have been engineered to express the IL-18 receptor complex (IL-18R and IL-18RAP) and induce an alkaline phosphatase reporter in response to IL-18, but not other cytokines or pathogen-associated molecular patterns. None of the IL-18 released by uninfected keratinocytes had activity, however, it was rendered active during GAS infection (Figure 2b). Next, we took cell-free supernatants from keratinocytes that contained this immature form of IL-18 and incubated it with GAS. GAS was able to again convert IL-18 into an active form (Figure 2c). Since IL-18 activation occurred in the absence of keratinocytes, we concluded that conventional cellular regulators (the inflammasome) were not essential, and instead, one or more GAS factors directly act on inert pro-IL-18 in a manner that renders it active.

\section{The GAS protease SpeB activates pro-IL-18}

In order to determine the bacterial determinants required for direct conversion of pro-IL-18 by GAS, we screened a panel of defined virulence factors mutants. During keratinocyte infection, we found that expression of the secreted cysteine protease SpeB was essential for GAS to convert IL-18 to an active form (Figure 3a). Consistent with this observation, CovRS, a two-component regulator of SpeB that is frequently 
inactivated in pathoadapted GAS that evade immune restriction, was also required for GAS conversion of IL-18 (Figure 3b). Plasmid complementation of $\triangle s p e B$ GAS 5448 restored activation, but plasmid encoding the catalytically inactive form $\mathrm{SpeB}_{\mathrm{C} 192 \mathrm{~S}}$ did not, indicating the protease activity of SpeB was required for IL-18 conversion (Figure 3c). We tested gain-of-function using Lactococcus lactis made to express SpeB and found that the catalytically active SpeB was necessary and sufficient for IL-18 activation (Figure 3d). Furthermore, in the absence of infection or other treatment, purified SpeB was sufficient to activate IL-18 (Figure 3e).

\section{pro-IL-18 is matured by SpeB cleavage}

IL-18 is conventionally activated when an N-terminal pro-domain (pro-IL-18) is proteolytically removed by Caspase-1 [22]. The truncated C-terminal portion then is freed from the steric hindrance that blocks binding and formation of a signaling complex with IL-18R and IL-18RAP [38]. In contrast to Caspase-1, which converted the $\sim 24 \mathrm{kDa}$ pro-IL-18 precursors to a $\sim 18 \mathrm{kDa}$ product, SpeB generated larger products spanning $\sim 22$ to $20 \mathrm{kDa}$ in size (Figure 4a). This is similar to IL-1 $\beta$, which SpeB cleaves at a different site than Caspase-1 to form a larger product that nonetheless is active [6]. However, there is no significant homology between the prodomains of IL-18 and IL-1 $\beta$ in mice or humans. The region proximal to the Caspase-1 cleavage site (after amino acid 36) of IL-18 makes contacts with the IL-18R in the co-crystal structure that stabilize binding and likely contribute to avidity and signaling [39]. Since the N-terminus occludes these interactions when attached [38], we were intrigued by how N-termini of different lengths generated by different protease cleavage sites impact IL-18 signaling (Figure $4 \mathbf{b}$ ). Thus, we next generated a series of $\mathrm{N}$-terminal truncations by in vitro transcription-translation to examine their activity with IL-18 reporter cells. Unlike IL-1 $\beta$, which similar techniques showed is only activated within a discrete internal region [40], truncation of as few as ten amino acids from the N-terminus of IL-18 was sufficient to significantly increase signaling (Figure 4c). Products smaller in size than those generated by Caspase-1 
rapidly loose activity, suggesting these residues are important in receptor interaction, but larger products like those generated by SpeB retained significant signaling activity when generated in recombinant forms (Figure 4c).

\section{SpeB-activated IL-18 induces inflammation}

IL-18 was originally discovered as a factor that induces type II interferon (IFN- $\gamma$ ) production from Th1 cells [41]. Since IL-18 is elevated in experimental murine infections as it is during human infection $[6,42,43]$, we decided to examine the role of IL-18 in a murine skin infection model. No difference in GAS growth was observed between wild-type C57B1/6 and $i l 18^{-/-}$mice (Figure 5a). Thus, irrespective of whether caspases, SpeB, or other mechanism activate IL-18, this model would be unable to capture whether that specific mechanism would have protective benefit. Nevertheless, IL-18 are IFN- $\gamma$ and critical in human immunity and are protective against infection [44-46]. Only a few instances have been identified where $i l 18^{-/-}$mice are susceptible to infection [19,47-49], suggesting differences in immune signaling between species or methods for modeling infection fail to capture IL-18-mediated signaling. IL-18 and T cell responses are instead commonly approached using human primary cells such whole blood or PBMCs to get around limitations imposed by mice $[43,50-53]$. Inhibition of IL-18 or IFN- $\gamma$ with neutralizing antibodies promoted GAS growth in whole human blood (Figure 5b). While this does not specifically show a role for keratinocyte-secreted IL-18 in the immune response, it supports that these cytokines can have a protective role of in the response of human immune cells to GAS, as they do for other pathogens.

PBMCs produce IFN- $\gamma$ in response to mature IL-18 when cultured with IL-12 [41]. Thus, we further examined whether IL-18 released by keratinocytes and processed by GAS is sufficient to induce IFN- $\gamma$ release in a co-culture model where keratinocytes are incubated with PBMCs and then infected with GAS

(Figure 5c). SpeB-expressing GAS induced significantly greater IFN- $\gamma$, and purified SpeB was sufficient to induce IFN- $\gamma$ (Figure 5d). IFN- $\gamma$ production correlated with the production of active IL-18 (Figure 5d). 
IL-18 was no longer fully SpeB-dependent as in keratinocyte monoculture (Figure 2b), but was nonetheless

157 significantly enhanced by SpeB (Figure 5d).

158

159

160

161

162

163

164

165

166

167

168

169

170

171

172

173

174

175 Discussion form.

Proteases are one of the most common protein classes and are frequently employed by pathogens as virulence factors. Like IL-18, IL-1 $\beta$ is directly cleaved and activated by bacterial proteases other than SpeB, such as Pseudomonas aeruginosa LasB [6,40]. These proteases potentially represent a group of "bacterial caspases" that may share in common the activation of these two related IL-1 family cytokines. We adapted our earlier models of keratinocyte infection (Figure $\mathbf{2 b}$ ) and cell-free processing of secreted pro-IL-18 (Figure 2c) to screened for species that can directly activate IL-18 (Figure 6a). We focused on a panel of Streptococci, many commonly reside on the human epithelia of the skin and upper respiratory tract, either as microbiota or pathogens, where they may contact keratinocyte-secreted pro-IL-18. During co-culture where live bacteria could infect live keratinocytes, nearly all species induced IL-18 activation by keratinocytes, which could proceed via conventional host processes, ie. the inflammasome (Figure 6b). However, only GAS and S. mutans significantly activated the inert pool of pro-IL-18 in cell-free supernatants collected from naïve keratinocytes (Figure 6b). This suggests constitutive pro-IL-18 secretion does not result in chronic inflammation in part because few proteases convert convert IL-18 into an active

SpeB is important for GAS colonization throughout the body and for penetrating deeper into tissue during invasive infection [6,54]. Skin keratinocytes are thus poised to mount some of the earliest immune responses against this important pathogen, but must safeguard against aberrant activation by the microbiota, 
since they are in constant contact with numerous other species they must tolerate. Consistent with prior observations [28-31], we observed constitutive pro-IL-18 secretion by primary human keratinocytes (Figure 1a, 2a, 2b), which is directly activated by SpeB (Figure 3e) to induce proinflammatory and antimicrobial responses (Figure 1a, 2a, 2b). Members of the healthy skin microbiota do not similarly activate immature pro-IL-18 (Figure 6b), so we hypothesize this pool of cytokine poised for activation serves as an early sentinel for infection by potentially invasive pathogens that make use of proteolytic virulence factors. By directly sensing specific proteases required for infection, IL-18 allows keratinocytes to discriminate between numerous species with high virulence potential and common pathogen-associated molecular patterns and toxins. While GAS is readily detected, low-level activation by the cariogenic opportunistic pathogen S. mutans suggests this mechanism could have importance in dental health. Furthermore, there may be pathogens from genera other than the Streptococcus with this activity, since proteases are broadly used as virulence factors by many human pathogens.

The mechanism underlying IL-18 discrimination of proteases remains unclear. Recent NMR studies show that mature (caspase-1-cleaved) IL-18 has a distinct spectra from pro-IL-18, suggesting that receptor binding is inhibited by intramolecular interactions from the pro-domain inducing major structural changes in the cytokine [38]. Amino-terminal truncations showed removal of the first 8 amino acids gave folding similar to pro-IL-18, the first 10 an intermediate form, and 12, 13, or 22 a form resembling the mature protein [38]. This closely coincides with our observations, where our mapping by IVTT observes partial activity starting with a 9 amino acid truncation (Figure 4c). Alternative endogenous proteases results in a variety of sizes [24-27]. Our observations, with those of Tsutsumi et al. [38], suggest that proteases that remove even a small portion of the inhibitory $\mathrm{N}$-terminus result in an active cytokine, whereas ones that generate smaller IL-18 forms may instead inactivate the cytokine. Nonetheless, the relatively loose substrate specificity of Pseudomonas LasB [40], with several potential cleavage sites in the N-terminus, did not result in cell-independent IL-18 activation (Figure 6b). Therefore, additional factors may regulate 
203 IL-18 activation beyond the primary sequence in this region, such as stabilizing reactions in the secondary 204 structure burying side chains recognized by LasB or other promiscuous proteases in the environment.

205 We further observed primary keratinocytes secrete a more diverse set of cytokines relative to 206 immortalized cell lines, including IL-18, a cytokine that serves as a crucial bridge from the innate immune 207 responses to the adaptive immune cells. However, all cytokine profiles were variable between cell lines, 208 which are commonly used or interpreted interchangeably. Autocrine or paracrine effects of these cytokines 209 should be considered when there is potential to impact experimental findings. Furthermore, antimicrobial 210 effectors are commonly co-regulated by many of the same signaling transduction networks, and these 211 potential defects in each line can potentially mask pathogen phenotypes. Altogether, we reiterate caution 212 should be exercised when immortalized cells are used for modeling host-pathogen interactions. Unlike 213 keratinocytes, endothelial cells did not release IL-18. Therefore, extracellular activation of IL-18 by SpeB 214 may not necessarily occur during sepsis or at distal sites from the skin surface, and may be most important 215 in priming responses early in infection. 


\section{Materials and Methods}

\section{Bacterial Strains}

220

GAS strain 5448, representative of the pandemic M1T1 clone, and its isogenic $\triangle s p e B, \Delta e m m 1, \Delta s l o, \Delta s p y A$, $\triangle c e p A, \triangle m a c, \Delta c o v R S$, and complemented pSpeB strains have been previously described $[6,35,55]$. GAS was statically grown at $37^{\circ} \mathrm{C}$ in Todd Hewitt broth (THB, Difco), washed two times with phosphate-buffered saline (PBS), and diluted to a multiplicity of infection (MOI) of 10 or 100 for in vitro infections. Selection for $\mathrm{pSpeB}$ was maintained with spectinomycin (Sigma) $100 \mathrm{ug} / \mathrm{ml}$ and expression controlled with titrations of anhydrotetracycline (Cayman Chemical) as previously [55]. The following reagent was obtained through BEI Resources, NIAID, NIH: Streptococcus anginosus F0211, Streptococcus cristatus F0329, Streptococcus downei F0415, Streptococcus equi ATCC 9528, Streptococcus gallolyticus TX20005, Streptococcus intermedius F0413, Streptococcus mitis F0392, Streptococcus parasanguinis F0449, and Streptococcus vestibularis F0396. Streptococcus mutans JH1140 was provided by S. McBride and Pseudomonas aeruginosa PA01 was used as previously described [40]. All additional strains are clinical isolates provided through the Emory University Investigational Clinical Microbiology Core. All bacteria were routinely propagated statically at $37^{\circ} \mathrm{C}$ in Todd Hewitt broth (Difco), washed two times with phosphate-buffered saline (PBS), and diluted to a multiplicity of infection (MOI) of 10 or 100 for in vitro infections.

\section{Cell Culture}

Primary human keratinocytes were supplied by PromoCell and cultured in Growth Medium 2 with supplement (C-20011, C-39011; PromoCell). Primary umbilical vein endothelial cells were supplied by Lifeline Cell Technology and cultured in endothelial cell growth medium (C-22010; PromoCell). A-431 lung epidermal cells (ATCC), Detroit 562 pharyngeal cells (ATCC), HaCat cells (unavailable from a repository, provided by C. Quave, Emory), and HEp-2 laryngeal cells (ATCC) were cultured in Eagle's 
Minimum Essential Medium (Gibco) supplemented with 10\% heat-inactivated fetal bovine serum (hiFBS, Atlanta Biologicals). HEK-Blue ${ }^{\text {TM }}$ IL-18 Reporter cells (Invivogen) were cultured in Dulbecco's Modified Eagle's Medium (Gibco) with 10\% hiFBS. $100 \mathrm{U} / \mathrm{mL}$ penicillin, $100 \mu \mathrm{g} / \mathrm{mL}$ streptomycin, and $100 \mu \mathrm{g} / \mathrm{mL}$ Normocin was supplemented during routine culture and omitted during experimental infections, unless otherwise noted. All cells were maintained at $37^{\circ} \mathrm{C}$ and $5 \% \mathrm{CO}_{2}$.

\section{Cytokine Measurements}

The relative levels of selected human cytokines and chemokines was determined in parallel using a membrane-based antibody array (ARY005B, R\&D Systems). Cells were seeded at $7.5 \times 10^{5}$ cells per well in a tissue-culture treated six-well plate. Cells were infected at an MOI of 10, and bacteria spun onto cells at $160 \mathrm{x} \mathrm{g}$ for three minutes. $1 \mathrm{~mL}$ of cell supernatant was collected after six hours of infection and incubated on the membrane overnight at $4^{\circ} \mathrm{C}$ and developed following the manufacturer's protocol. Chemiluminescence was measured using the ChemiDoc MP imaging system, and mean pixel density was quantified using ImageJ. Only proinflammatory cytokines made by at least one of the cells lines under study are presented graphically. IL-18 signaling activity was measured in $50 \mu \mathrm{L}$ volumes of cell supernatant using HEK-Blue ${ }^{\mathrm{TM}}$ IL-18 Reporter cells (Invivogen) and secreted alkaline phosphatase activity measured after $18 \mathrm{~h}$ incubation as previously [56]. This was normalized total IL-18 measured by ELISA (R\&D Systems).

\section{Protein Purification}

The fully spliced coding sequence for human $i l 18$ and murine $i l 18$, encoding pro-IL-18 for each species, was generated by gene synthesis for expression from pETPP [35] to generate pETPP-hIL18 and pETPPmIL18. Expression was induced from the plasmids in BL21 cell induced with $0.2 \mathrm{mM}$ IPTG (Sigma) overnight at $18^{\circ} \mathrm{C}$. Bacterial cell pellets were suspended in $10 \mathrm{~mL}$ of phosphate buffered saline (PBS, $\mathrm{pH}$ 
7.4). Cells were completely lysed by sonication at $40 \%$ amplitude for two minutes for 30 seconds at 10

267 second intervals and centrifugation at $7500 \mathrm{x}$ g for 10 minutes. Lysate was through Talon gravity columns

268 loaded with HisPur ${ }^{\mathrm{TM}}$ Cobalt Resin (Thermo Scientific), washed with PBS, and eluted in PBS supplemented

269 with $300 \mathrm{mM}$ imidazole. SpeB was purified as previously [6]. Cleavage of purified human and murine pro-

270 IL-18 was performed as previously with pro-IL-1 $[6]$, with SpeB (100 ng) and Caspase-1 (1U, Novus) in

271 PBS with $2 \mathrm{mM}$ dithiothreitol $2 \mathrm{~h}$ at $37{ }^{\circ} \mathrm{C}$. Reactions were stopped by the addition Laemmli buffer, $10 \%$

$272 \beta$-ME, and $1 \times$ SDS loading buffer solution, then boiled at $95^{\circ} \mathrm{C}$ for $5 \mathrm{~min}$. Samples were analyzed by SDS-

273 PAGE on Tris-Glycine gels (Invitrogen) and visualized with AquaStain (Bulldog Bio).

274

\section{In vitro transcription/translation}

IL-18 truncations were generated through in vitro transcription/translation using pET-pro-IL-18 as a template in $10 \mu \mathrm{l}$ reaction volumes following the manufacturer's recommendations (TNT Coupled

Reticulocyte Lysate; Promega) as previously [40], with 5' primers gaaattaatacgactcactatagggagaccccacc-

(IVTTIL18-9), gaaattaatacgactcactatagggagaccccaccatggcttttgtggcaatgaaattta (IVTTIL18-13), gaaattaatacgactcactatagggagaccccaccatggcttttattgacaatacgcttta (IVTTIL18-18), gaaattaatacgactcactatagggagaccccaccatggctaaatttattgacaatacgct (IVTTIL18-17), gaaattaatacgactcactatagggagaccccaccatggctaatacgctttactttatagc (IVTTIL18-21), gaaattaatacgactcactatagggagaccccaccatggcttttatagctgaagatgatg (IVTTIL18-25), gaaattaagacccaccatggctctggaatcagattactttg (IVTTIL18-33), gaaattaatacgactcactatagggagaccccaccatggcttactttggcaagcttgaat (IVTTIL18-37), gaaattaatacgactcactatagggagaccccaccatggctcttgaatctaaattatcag (IVTTIL1841), gaaattaatacgactcactatagggagaccccaccatggctataagaaatttgaatgacc (IVTTIL18-48), gaaattaatacgactcactatagggagaccccaccatggctattgaccaaggaaatcggc (IVTTIL18-58), in combination with 3 , primer 
total IL-18 measured by ELISA (R\&D Systems) following manufacturer protocol.

\section{Ethics Statement}

294 This study was conducted according to the principles expressed in the Declaration of Helsinki. Blood was

295 collected from healthy adult volunteers under informed consent and approved by the Institutional Review

296 Board of Emory University. Animal experiments were approved by the Institutional Animal Care and Use

297 Committees of Emory University.

298

299 Statistics and Data Analysis

300 Values are expressed as mean \pm standard deviation. Differences between groups were analyzed using 1301 way analysis of variance with Dunnett multiple comparisons analysis unless otherwise indicated. 302 Differences are considered statistically significant at a P value of $<.05$ using GraphPad Prism v9 software. 303 Principal Component Analysis (PCA) was used to reduce the number of variables needed to adequately 304 describe differences in cytokine profiles; each condition was treated as an independent variable and 305 multivariate analysis was performed in Prism v9. Protein models were visualized using PyMol 2.3.3. 306 Diagrams created with BioRender.com.

\section{Acknowledgements}

309 We thank Victor Nizet for his insights and bacteria, all members of the LaRock lab for discussions, 310 Cassandra Quave for cell lines, the Children's Healthcare of Atlanta and Emory University's Children's 311 Clinical and Translational Discovery Core (CTDC) for whole blood and processing, Shonna McBride, BEI 312 Resources, and the Emory Investigational Clinical Microbiology Core (ICMC) for bacterial isolates. 
315 Funding

316 The CTDC received support from Children's Healthcare of Atlanta and Emory University, the ICMC from

317 the Emory Department of Medicine, Division of Infectious Diseases, and C.N.L. from National Institutes

318 of Health grants AI130223 and AI153071. No funders contributed to the study design or conclusions.

320 Author contributions

321 C.N.L. conceived this study, C.N.L., A.F.J., and J.S.S. designed the experiments and wrote the paper.

322 A.F.J., J.S.S., K.T., R.R., D.L.L., and C.N.L. performed experiments and analyzed results.

\section{Competing Interests}

325 The authors declare that no competing interests exist.

326

\section{Correspondence}

328 Correspondence and requests for materials should be addressed to: christopher.larock@emory.edu 


\section{REFERENCES}

332

333

334

335

336

337

338

339

340

341

342

343

344

345

346

347

348

349

350

351

352

353

354

355

356

357

358

359

360

361

362

363

364

365

1. Okada N, Liszewski MK, Atkinson JP, Caparon M. Membrane cofactor protein (CD46) is a keratinocyte receptor for the M protein of the group A streptococcus. Proc Natl Acad Sci U S A. 1995;92: 2489-2493.

2. Soderholm AT, Barnett TC, Korn O, Rivera-Hernandez T, Seymour LM, Schulz BL, et al. Group A Streptococcus M1T1 Intracellular Infection of Primary Tonsil Epithelial Cells Dampens Levels of Secreted IL-8 Through the Action of SpyCEP. Front Cell Infect Microbiol. 2018;8: 160. doi:10.3389/fcimb.2018.00160

3. Darmstadt GL, Mentele L, Podbielski A, Rubens $C$. Role of group A streptococcal virulence factors in adherence to keratinocytes. Infect Immun. 2000;68: 1215-1221. doi:10/cg6pm2

4. Schrager HM, Rheinwald JG, Wessels MR. Hyaluronic acid capsule and the role of streptococcal entry into keratinocytes in invasive skin infection. J Clin Invest. 1996;98: 1954-1958.

doi:10.1172/JCl118998

5. Ralph AP, Carapetis JR. Group A Streptococcal Diseases and Their Global Burden. Curr Top Microbiol Immunol. 2012; 1-27. doi:10/gjmhkr

6. LaRock CN, Todd J, LaRock DL, Olson J, O'Donoghue AJ, Robertson AAB, et al. IL-1ß is an innate immune sensor of microbial proteolysis. Sci Immunol. 2016;1: eaah3539.

doi:10.1126/sciimmunol.aah3539

7. Shannon BA, McCormick JK, Schlievert PM. Toxins and Superantigens of Group A Streptococci. Microbiol Spectr. 2019;7. doi:10.1128/microbiolspec.GPP3-0054-2018

8. Wilde S, Olivares KL, Nizet V, Hoffman HM, Radhakrishna S, LaRock CN. Opportunistic Invasive Infection by Group A Streptococcus During Anti-Interleukin-6 Immunotherapy. J Infect Dis. 2021;223: 1260-1264. doi:10/gg8f36

9. Kasper KJ, Zeppa JJ, Wakabayashi AT, Xu SX, Mazzuca DM, Welch I, et al. Bacterial Superantigens Promote Acute Nasopharyngeal Infection by Streptococcus pyogenes in a Human MHC Class IIDependent Manner. PLoS Pathog. 2014;10: e1004155. doi:10.1371/journal.ppat.1004155

10. Nakamura K, Okamura H, Nagata $\mathrm{K}$, Komatsu T, Tamura T. Purification of a factor which provides a costimulatory signal for gamma interferon production. Infect Immun. 1993;61: 64-70. doi:10/gg79qw

11. Gu Y, Kuida K, Tsutsui H, Ku G, Hsiao K, Fleming MA, et al. Activation of interferon-gamma inducing factor mediated by interleukin-1beta converting enzyme. Science. 1997;275: 206-209.

12. Ghayur T, Banerjee S, Hugunin M, Butler D, Herzog L, Carter A, et al. Caspase-1 processes IFNgamma-inducing factor and regulates LPS-induced IFN-gamma production. Nature. 1997;386: 619623. 
13. Hoshino K, Tsutsui H, Kawai T, Takeda K, Nakanishi K, Takeda Y, et al. Cutting edge: generation of IL18 receptor-deficient mice: evidence for IL-1 receptor-related protein as an essential IL-18 binding receptor. J Immunol. 1999;162: 5041-5044.

14. Raupach B, Peuschel S-K, Monack D, Zychlinsky A. Caspase-1-mediated activation of interleukin1 beta (IL-1beta) and IL-18 contributes to innate immune defenses against Salmonella enterica serovar Typhimurium infection. Infect Immun. 2006;74: 4922-4926. doi:10.1128/IAI.00417-06

15. Sansonetti PJ, Phalipon A, Arondel J, Thirumalai K, Banerjee S, Akira S, et al. Caspase-1 Activation of IL-1 $\beta$ and IL-18 Are Essential for Shigella flexneri-Induced Inflammation. Immunity. 2000;12: 581590. doi:10/cmg87h

16. Bohn E, Sing A, Zumbihl R, Bielfeldt C, Okamura H, Kurimoto M, et al. IL-18 (IFN- $\gamma$-Inducing Factor) Regulates Early Cytokine Production in, and Promotes Resolution of, Bacterial Infection in Mice. J Immunol. 1998;190: 10.

17. Tsuji NM, Tsutsui H, Seki E, Kuida K, Okamura H, Nakanishi K, et al. Roles of caspase-1 in Listeria infection in mice. Int Immunol. 2004;16: 335-343. doi:10/fgfxjx

18. Ceballos-Olvera I, Sahoo M, Miller MA, Del Barrio L, Re F. Inflammasome-dependent pyroptosis and IL-18 protect against Burkholderia pseudomallei lung infection while IL-1 $\beta$ is deleterious. PLoS Pathog. 2011;7: e1002452. doi:10.1371/journal.ppat.1002452

19. Sugawara I, Yamada H, Kaneko H, Mizuno S, Takeda K, Akira S. Role of Interleukin-18 (IL-18) in Mycobacterial Infection in IL-18-Gene-Disrupted Mice. Infect Immun. 1999;67: 2585-2589.

20. Lauw FN, Branger J, Florquin S, Speelman P, Van Deventer SJH, Akira S, et al. IL-18 improves the early antimicrobial host response to pneumococcal pneumonia. J Immunol. 2002;168: 372-378.

21. Cusumano V, Midiri A, Cusumano VV, Bellantoni A, De Sossi G, Teti G, et al. Interleukin-18 is an essential element in host resistance to experimental group $B$ streptococcal disease in neonates. Infect Immun. 2004;72: 295-300.

22. LaRock CN, Cookson BT. Burning Down the House: Cellular Actions during Pyroptosis. PLoS Pathog. 2013;9: e1003793. doi:10.1371/journal.ppat.1003793

23. Bossaller L, Chiang P-I, Schmidt-Lauber C, Ganesan S, Kaiser WJ, Rathinam VAK, et al. Cutting edge: FAS (CD95) mediates noncanonical IL-1 $\beta$ and IL-18 maturation via caspase-8 in an RIP3independent manner. J Immunol. 2012;189: 5508-5512. doi:10.4049/jimmunol.1202121

24. Omoto Y, Yamanaka K, Tokime K, Kitano S, Kakeda M, Akeda T, et al. Granzyme B is a novel interleukin-18 converting enzyme. J Dermatol Sci. 2010;59: 129-135. doi:10.1016/j.jdermsci.2010.05.004

25. Omoto Y, Tokime K, Yamanaka K, Habe K, Morioka T, Kurokawa I, et al. Human mast cell chymase cleaves pro-IL-18 and generates a novel and biologically active IL-18 fragment. J Immunol Baltim Md 1950. 2006;177: 8315-8319. doi:10.4049/jimmunol.177.12.8315 
401

402

403

404

405

406

407

408

409

410

411

412

413

414

415

416

417

418

419

420

421

422

423

424

425

426

427

428

429

430

431

432

433

434

435

436

26. Sugawara S, Uehara A, Nochi T, Yamaguchi T, Ueda H, Sugiyama A, et al. Neutrophil proteinase 3mediated induction of bioactive IL-18 secretion by human oral epithelial cells. J Immunol. 2001;167: 6568-6575.

27. Robertson SE, Young JD, Kitson S, Pitt A, Evans J, Roes J, et al. Expression and alternative processing of IL-18 in human neutrophils. Eur J Immunol. 2006;36: 722-731. doi:10/bs4ht7

28. Companjen AR, van der Velden VH, Vooys A, Debets R, Benner R, Prens EP. Human keratinocytes are major producers of IL-18: predominant expression of the unprocessed form. Eur Cytokine Netw. 2000;11: 383-390.

29. Mee JB, Alam Y, Groves RW. Human keratinocytes constitutively produce but do not process interleukin-18. Br J Dermatol. 2000;143: 330-336. doi:10.1046/j.1365-2133.2000.03759.x

30. Mizutani H, Black RA, Kupper T. Human keratinocytes produce but do not process pro-interleukin-1 (IL-1) beta. Different strategies of IL-1 production and processing in monocytes and keratinocytes. J Clin Invest. 1991;87: 1066-1071.

31. Korhonen E, Piippo N, Hytti M, Hyttinen JMT, Kaarniranta K, Kauppinen A. Only IL-1 $\beta$ release is inflammasome-dependent upon ultraviolet B irradiation although IL-18 is also secreted. FASEB J. 2020;34: 6437-6448. doi:10.1096/fj.201902355RR

32. Wang B, Ruiz N, Pentland A, Caparon M. Keratinocyte Proinflammatory Responses to Adherent and Nonadherent Group A Streptococci. Infect Immun. 1997;65: 2119-2126.

33. Persson ST, Wilk L, Morgelin M, Herwald H. Vigilant Keratinocytes Trigger Pathogen-Associated Molecular Pattern Signaling in Response to Streptococcal M1 Protein. Infect Immun. 2015;83: 4673-4681. doi:10.1128/IAI.00887-15

34. Ruiz N, Wang B, Pentland A, Caparon M. Streptolysin O and adherence synergistically modulate proinflammatory responses of keratinocytes to group A streptococci. Mol Microbiol. 1998;27: 337346.

35. LaRock CN, Döhrmann S, Todd J, Corriden R, Olson J, Johannssen T, et al. Group A Streptococcal M1 Protein Sequesters Cathelicidin to Evade Innate Immune Killing. Cell Host Microbe. 2015; 1-9. doi:10.1016/j.chom.2015.09.004

36. Walker MJ, Hollands A, Sanderson-Smith ML, Cole J, Kirk JK, Henningham A, et al. DNase Sda1 provides selection pressure for a switch to invasive group A streptococcal infection. Nat Med. 2007;13: 981-985.

37. Flaherty RA, Donahue DL, Carothers KE, Ross JN, Ploplis VA, Castellino FJ, et al. Neutralization of Streptolysin S-Dependent and Independent Inflammatory Cytokine IL-1 $\beta$ Activity Reduces Pathology During Early Group A Streptococcal Skin Infection. Front Cell Infect Microbiol. 2018;8: e57285. doi:10.3389/fcimb.2018.00211

38. Tsutsumi N, Yokota A, Kimura T, Kato Z, Fukao T, Shirakawa M, et al. An innate interaction between IL-18 and the propeptide that inactivates its precursor form. Sci Rep. 2019;9: 6160. doi:10/gf5znd 
39. Tsutsumi N, Kimura T, Arita K, Ariyoshi M, Ohnishi H, Yamamoto T, et al. The structural basis for receptor recognition of human interleukin-18. Nat Commun. 2014;5: 5340. doi:10.1038/ncomms6340

40. Sun J, LaRock DL, Skowronski EA, Kimmey JM, Olson J, Jiang Z, et al. The Pseudomonas aeruginosa protease LasB directly activates IL-1B. EBioMedicine. 2020;60. doi:10/gjmhkt

41. Okamura $\mathrm{H}$, Tsutsuit $\mathrm{H}$, Komatsut $\mathrm{T}$, Yutsudo $\mathrm{M}$, li TT, li KT, et al. Cloning of a new cytokine that induces IFN-y production by T cells. Nature. 1995;378: 88-91.

42. Wang S-M, Lu I-H, Lin Y-L, Lin Y-S, Wu J-J, Chuang W-J, et al. The severity of Streptococcus pyogenes infections in children is significantly associated with plasma levels of inflammatory cytokines. Diagn Microbiol Infect Dis. 2008;61: 165-169. doi:10.1016/j.diagmicrobio.2008.01.008

43. Dan JM, Havenar-Daughton C, Kendric K, Al-kolla R, Kaushik K, Rosales SL, et al. Recurrent group A Streptococcus tonsillitis is an immunosusceptibility disease involving antibody deficiency and aberrant TFH cells. Sci Transl Med. 2019;11: eaau3776. doi:10.1126/scitranslmed.aau3776

44. Zhou T, Damsky W, Weizman O-E, McGeary MK, Hartmann KP, Rosen CE, et al. IL-18BP is a secreted immune checkpoint and barrier to IL-18 immunotherapy. Nature. 2020; 1-6. doi:10.1038/s41586020-2422-6

45. Brydges SD, Broderick L, McGeough MD, Pena CA, Mueller JL, Hoffman HM. Divergence of IL-1, IL18, and cell death in NLRP3 inflammasomopathies. J Clin Invest. 2013;123: 4695-4705. doi:10.1172/JCl71543

46. Vecchié A, Bonaventura A, Toldo S, Dagna L, Dinarello CA, Abbate A. IL-18 and infections: Is there a role for targeted therapies? J Cell Physiol. 2020; 1-20. doi:10.1002/jcp.30008

47. Mancuso G, Midiri A, Beninati C, Zummo S, Biondo C. Protective role of IL-18 in host defenses against group B Streptococcus. Eur J Clin Microbiol Infect Dis. 2021. doi:10.1007/s10096-02104299-y

48. Mitchell AJ, Yau B, McQuillan JA, Ball HJ, Too LK, Abtin A, et al. Inflammasome-dependent IFN- $\gamma$ drives pathogenesis in Streptococcus pneumoniae meningitis. J Immunol. 2012;189: 4970-4980. doi:10.4049/jimmunol.1201687

49. Von Moltke J, Ayres JS, Kofoed EM, Chavarria-Smith J, Vance RE. Recognition of Bacteria by Inflammasomes. Annu Rev Immunol. 2013;31: 73-106.

50. Emgård J, Bergsten H, McCormick JK, Barrantes I, Skrede S, Sandberg JK, et al. MAIT Cells Are Major Contributors to the Cytokine Response in Group A Streptococcal Toxic Shock Syndrome. Proc Natl Acad Sci. 2019;116: 25923-25931.

51. Wilde S, Johnson AF, LaRock CN. Playing With Fire: Proinflammatory Virulence Mechanisms of Group A Streptococcus. Front Cell Infect Microbiol. 2021;11: 704099. doi:10/gk4rc8 
52. Meilleur CE, Wardell CM, Mele TS, Dikeakos JD, Bennink JR, Mu H-H, et al. Bacterial Superantigens Expand and Activate, Rather than Delete or Incapacitate, Preexisting Antigen-Specific Memory CD8+ T Cells. J Infect Dis. 2019;219: 1307-1317. doi:10/gf54ps

53. Shaler CR, Choi J, Rudak PT, Memarnejadian A, Szabo PA, Tun-Abraham ME, et al. MAIT cells launch a rapid, robust and distinct hyperinflammatory response to bacterial superantigens and quickly acquire an anergic phenotype that impedes their cognate antimicrobial function: Defining a novel mechanism of superantigen-induced immunopathology and immunosuppression. PLoS Biol. 2017;15: e2001930. doi:10.1371/journal.pbio.2001930

54. Nelson DC, Garbe J, Collin M. The cysteine proteinase SpeB from Streptococcus pyogenes- a potent modifier of immunologically important host and bacterial proteins. Biol Chem. 2011;392: 10771088. doi:10.1515/BC-2011-208

55. Barnett TC, Liebl D, Seymour LM, Gillen CM, Lim JY, LaRock CN, et al. The Globally Disseminated M1T1 Clone of Group A Streptococcus Evades Autophagy for Intracellular Replication. Cell Host Microbe. 2013;14: 675-682.

56. LaRock DL, Sands JS, Ettouati E, Richard M, Bushway PJ, Adler ED, et al. Inflammasome inhibition blocks cardiac glycoside cell toxicity. J Biol Chem. 2019;294: 12846-12854. 
A
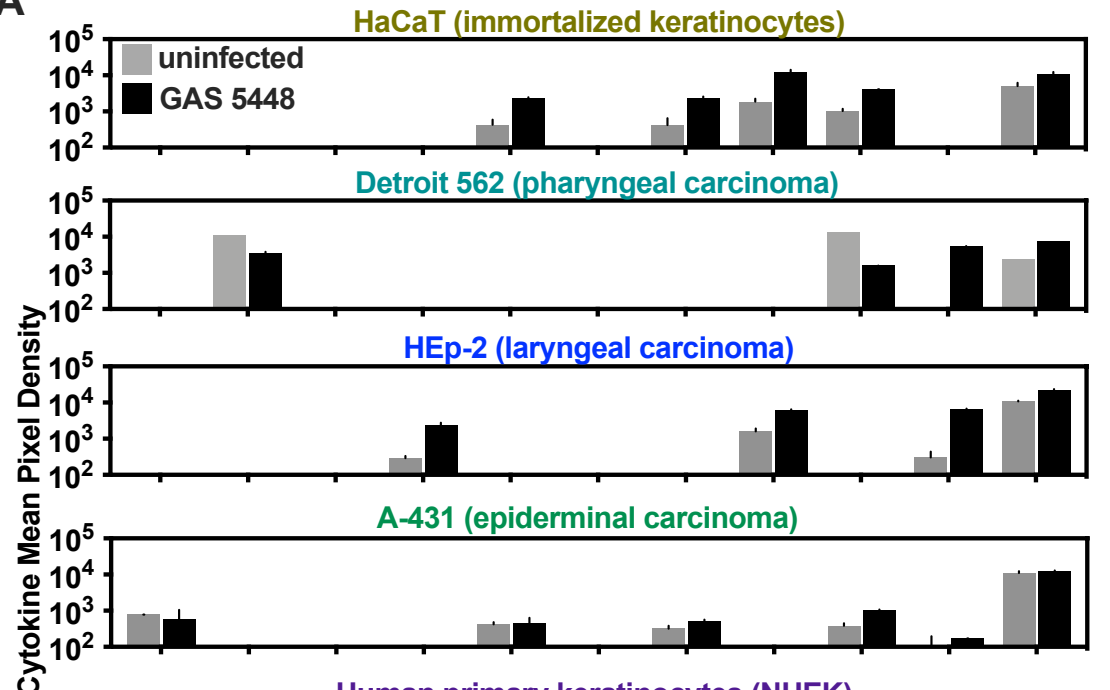

जे
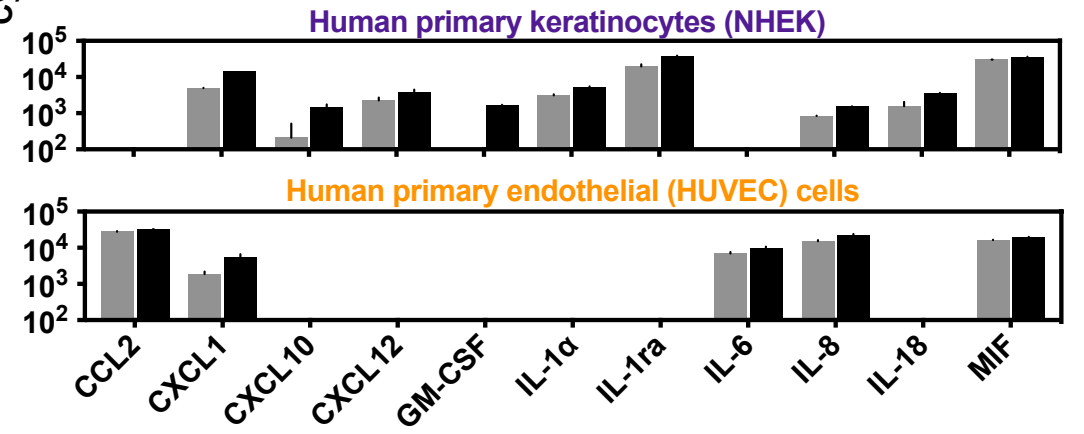

B
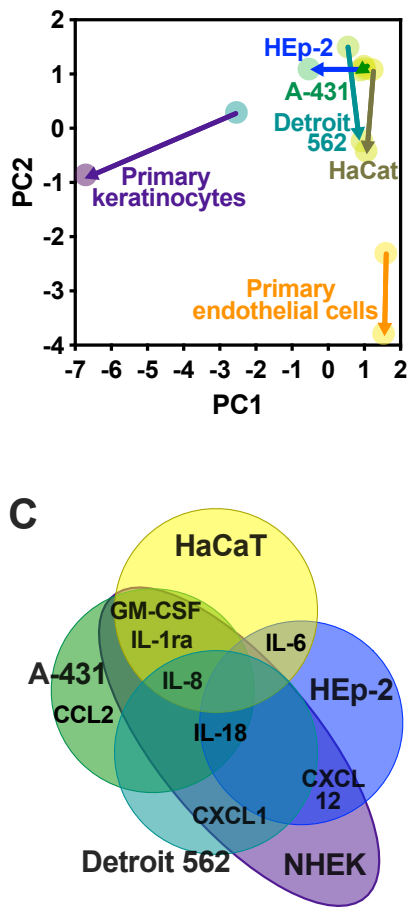

489

490

491

492

493

494

495

496

497

Figure 1. Cytokine profiles of keratinocytes and related cell lines. HaCaT, Detroit 562, HEp-2, A-431, primary keratinocytes, or HUVEC cells were infected with $7.5 \times 10^{6}$ colony forming units (CFU) of GAS for 6 h. (A) Relative abundance of select cytokines was examined by membrane-based antibody array. (B) Cytokine profiles of each cell were examined by multivariate (principal component analysis). Arrows indicate change in cells from uninfected to $6 \mathrm{~h}$ infection. (C) Graphical representation of the congruent cytokine profiles between cell types. 

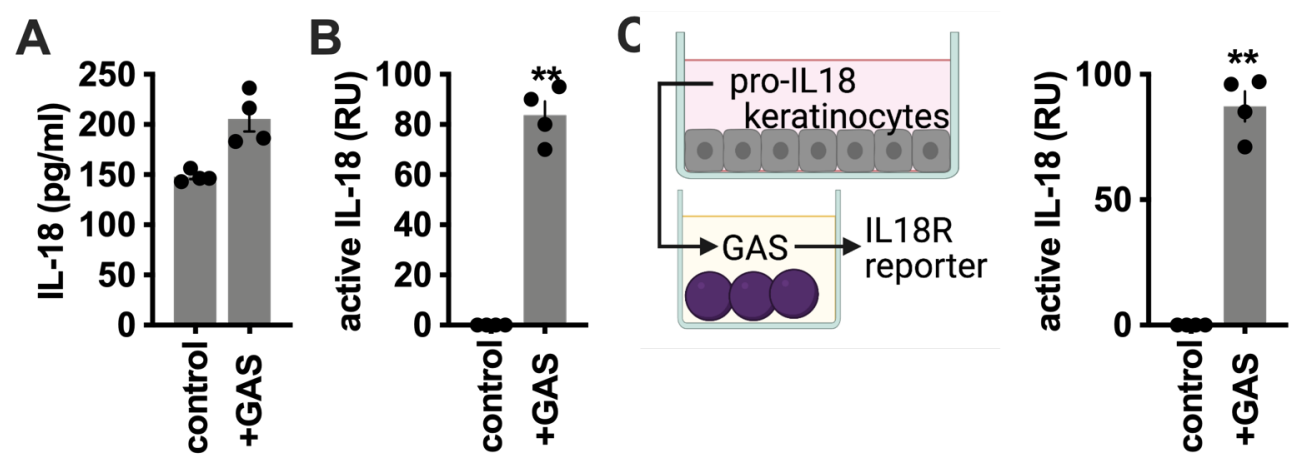

Figure 2. Examination of IL-18 activation in GAS-infected keratinocytes. Primary human keratinocytes were infected with GAS at a multiplicity of infection (MOI) of 10 for $4 \mathrm{~h}$. (A) Secreted IL-18 (total, proand mature forms) was measured by ELISA. (B) Bioactive IL-18, capable of inducing signaling through the IL-18R/IL-18RAP receptor complex, was measured with HEK-Blue IL18 reporter cells. (C) Supernatants removed from primary human keratinocytes were incubated with GAS and IL-18 activity measured as in (B). Data were analyzed by 1-way ANOVA using Dunnett multiple comparisons analysis. All data represent at least 3 independent experiments with 4 replicates. Bars show median values \pm standard deviation. $* * P<.005$; ns, not significant. 
A

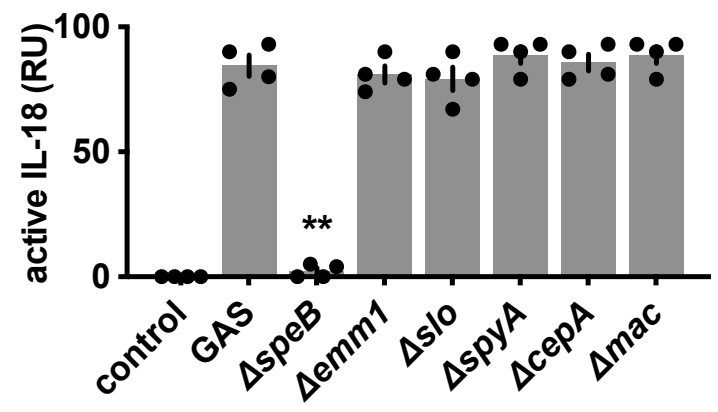

B

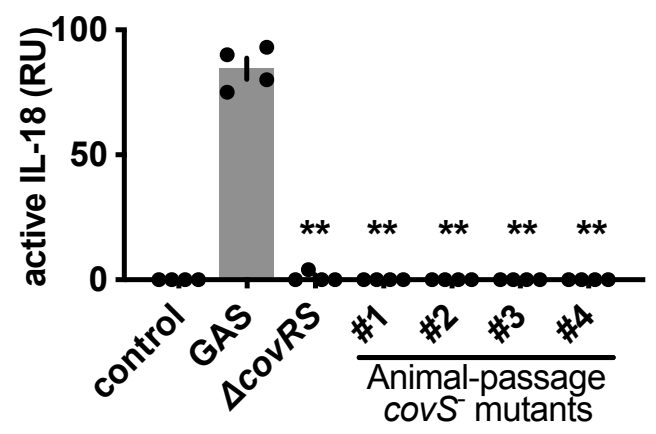

C

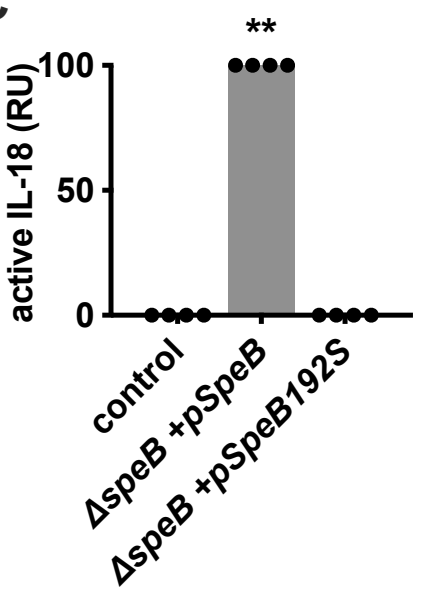

D

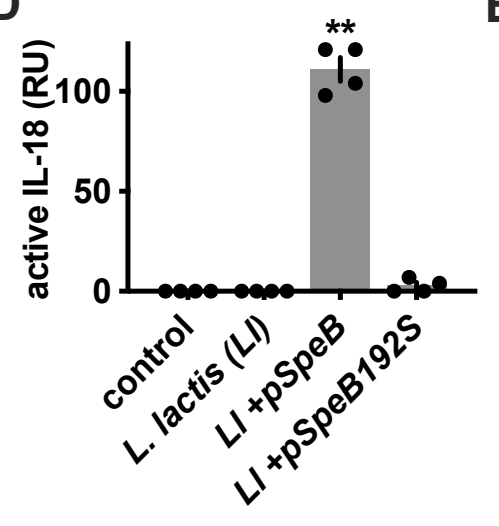

E

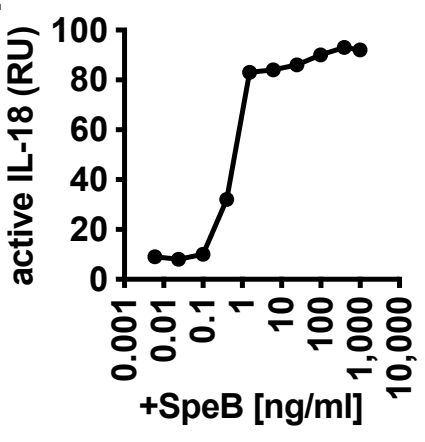

Figure 3. Examination of GAS requirements for IL-18 activation. Primary human keratinocytes were infected with GAS at MOI 10 for 4 h, then bioactive IL-18 was measured with HEK-Blue IL-18 reporter cells. (A and B) IL-18 activation was measured in the supernatants from keratinocytes infected with the indicated gene knockouts of GAS strain 5448. (C) IL-18 activation was measured in the supernatants from keratinocytes incubated with spectinomycin and anhydrotetracycline to maintain SpeB expression during infection with $\triangle s p e B$ GAS 5448 carrying the indicated plasmids. (D) IL-18 activation was measured in the supernatants from keratinocytes during infection with L. lactis, spectinomycin and anhydrotetracycline were included during infections with the plasmid-carrying strains. (E). IL-18 activation was measured in the supernatants from keratinocytes treated with titrations of purified SpeB protein. Data were analyzed by 1-way ANOVA using Dunnett multiple comparisons analysis. All data represent at least 3 independent experiments with 4 replicates. Bars show median values \pm standard deviation. ${ }^{* *} P<.005$; ns, not significant. 

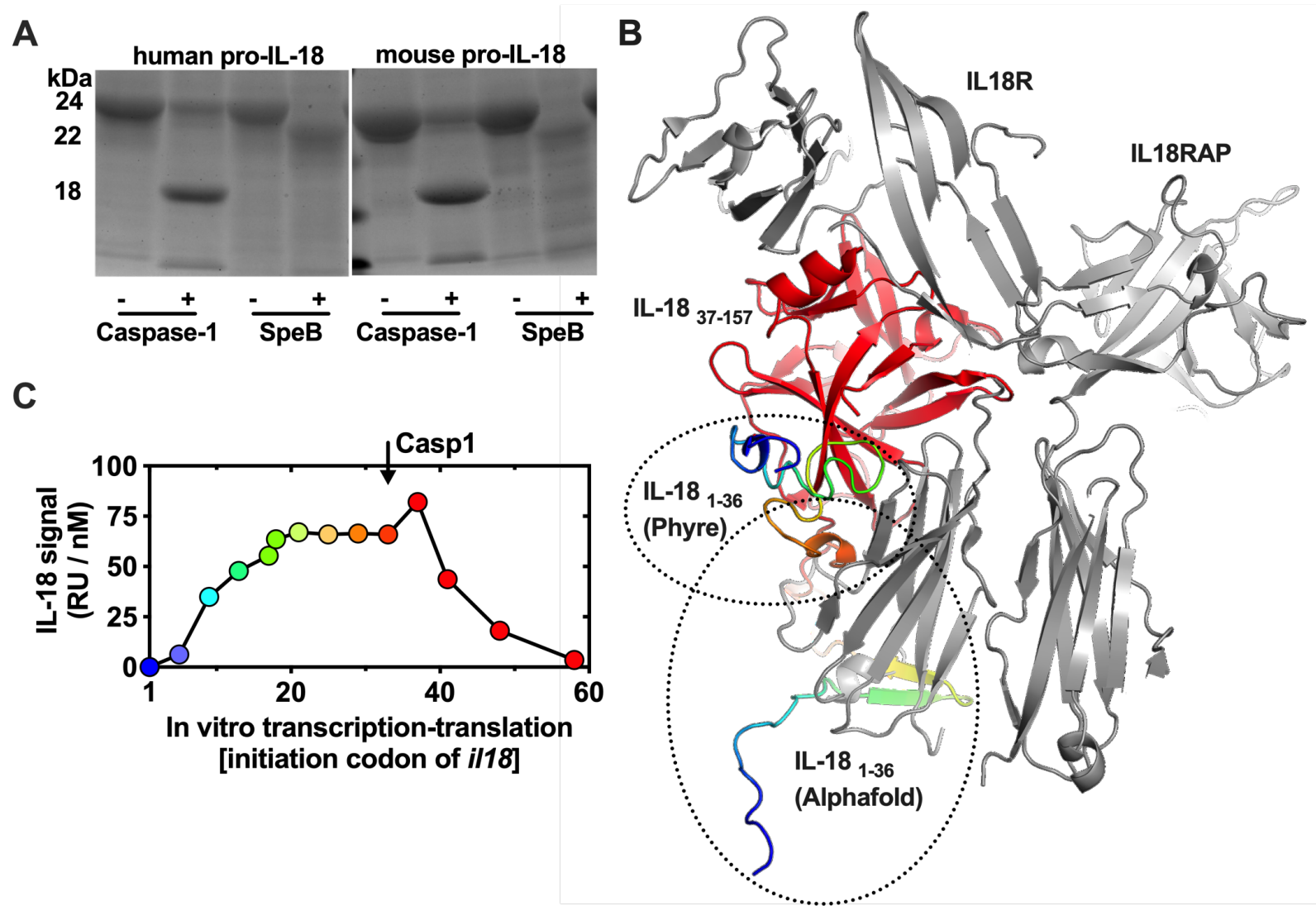

Figure 4. Examination of SpeB processing of IL-18. (A) Recombinant human and murine pro-IL-18 was purified and incubated with purified, active human Caspase- 1 or purified, active SpeB, then cleavage products separated by SDS-PAGE and visualized by staining. (B) Structure of the human IL-18 receptor complex (PDB:3wo4; [39]), IL18R and IL18RAP; grey, residues 37-157 of IL18 (caspase-cleaved product); red, predicted N-terminal (residues 1-36) inhibitor domain; rainbow. (C) Signaling activity of recombinant IL-18 N-terminal truncations generated using in vitro transcription-translation from the human ill 8 gene with coding beginning at the indicated codon, 1 is full-length pro-IL-18; colors match positions in (B). Activity toward HEK-Blue IL18 reporter cells was normalized for loading by quantifying total product using IL-18-specific ELISA. 

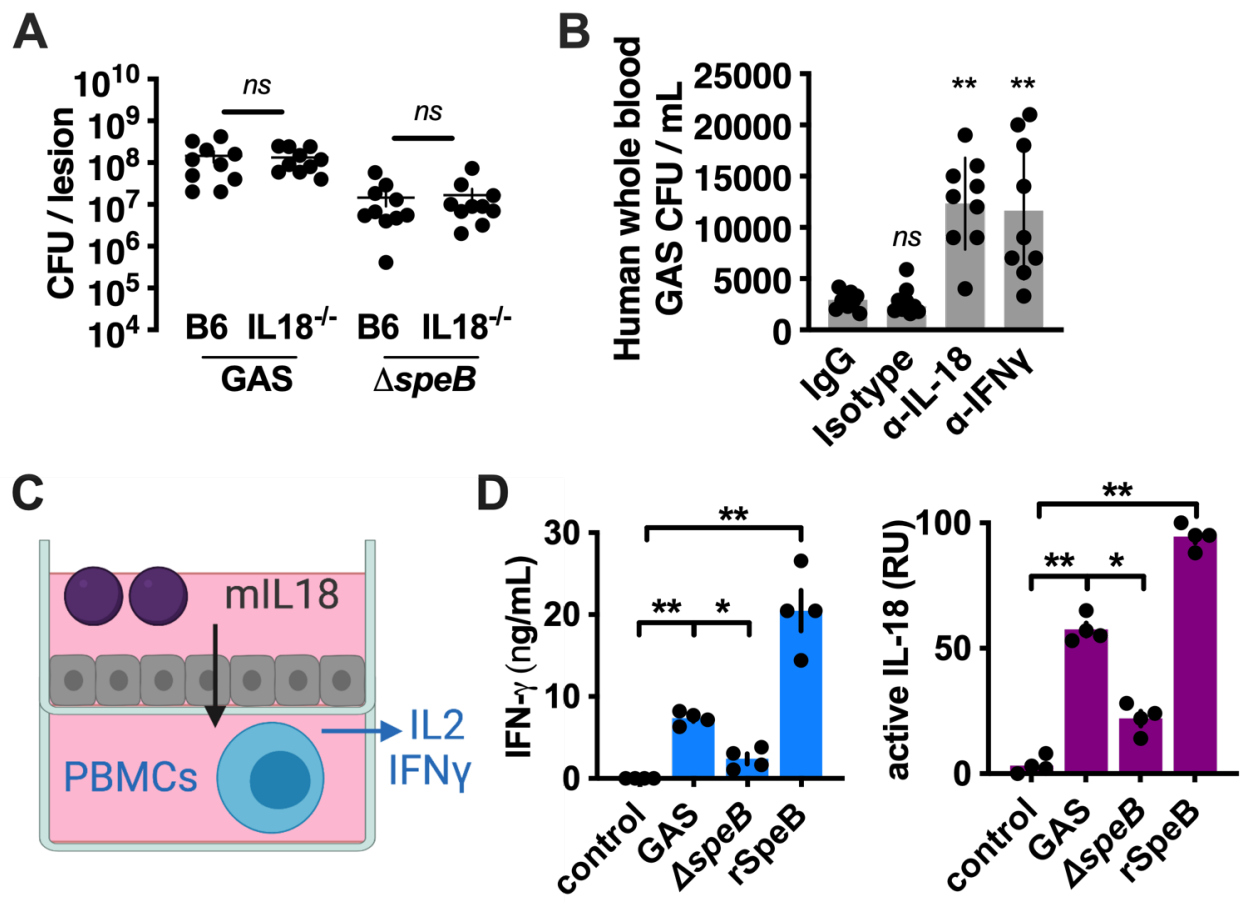

Figure 5. SpeB activation of IL-18 promotes antimicrobial IFN- $\gamma$ responses. (A) C57BL/6 wild-type or IL18-knockout (IL18 $8^{-/-}$) mice were inoculated intradermally with $10^{8}$ CFU of GAS 5448 or its $\triangle$ speB mutant. After $72 \mathrm{~h}$, mice were euthanized and GAS CFU enumerated at the infection site. Results are from 2 independent experiments with 5 mice in each. (B) Heparinized whole human blood treated with isotype antibody control, $\alpha$-IL-18, or $\alpha$-IFN- $\gamma$ neutralizing antibodies $(50 \mu \mathrm{g} / \mathrm{ml})$ was inoculated with $10^{5} \mathrm{CFU}$ of GAS 5448 and CFU enumerated after $4 \mathrm{~h}$. Results are pooled from 3 independent experiments. (C) Diagram of primary keratinocyte/PBMC co-culture model; IFN- $\gamma$ is a reporter of T cell activation, which can occur via IL-18 and additional mechanisms during infection. (D) Cocultured keratinocyte/PBMCs were infected with $10^{5} \mathrm{CFU}$ of GAS or treated with rSpeB. After $4 \mathrm{~h}$, active IL-18 was quantified with HEK-Blue IL-18 reporter cells, and IFN- $\gamma$ by ELISA. Data represent at least 3 independent experiments with 4 replicates. Data were analyzed by 1-way ANOVA using Dunnett multiple comparisons analysis. Bars show median values \pm standard deviation. $* P<.05 ; * * P<.005 ;$ ns, not significant. 
A

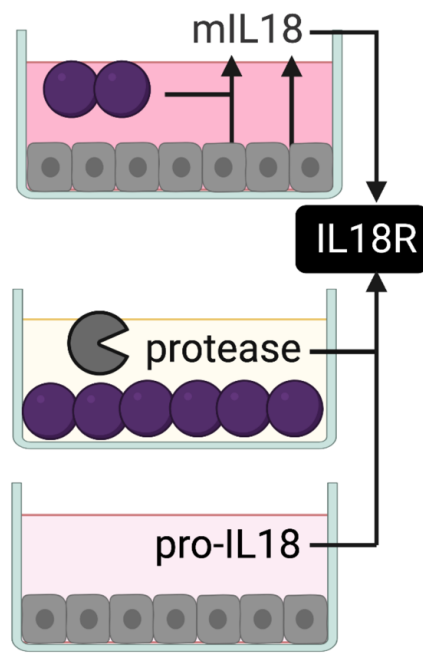

B

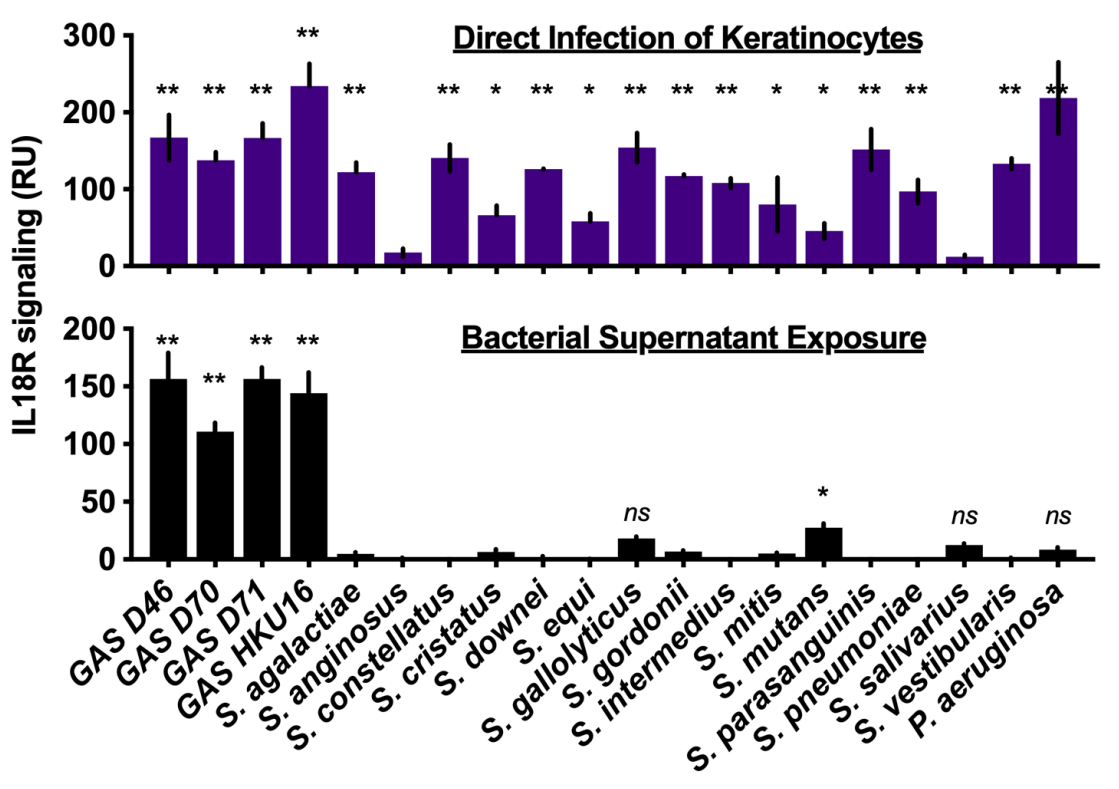

Figure 6. Bacterial activation of IL18. (A) Diagram of infection model. (B) In 'Direct Infection of Keratinocytes' primary human keratinocytes were infected with each bacteria at MOI 10. In 'Bacterial Supernatant Exposure', an equivalent volume of overnight culture supernatant was combined with cell-free supernatants from keratinocyte ex vivo. After $4 \mathrm{~h}$, for both models, bioactive IL-18 was measured with HEK-Blue IL18 reporter cells. Data represent at least 3 independent experiments with 4 replicates. Data were analyzed by 1-way ANOVA using Dunnett multiple comparisons analysis compared to uninfected/untreated keratinocytes. Bars show median values \pm standard deviation. $* P<.05 ; * * P<.005$; ns, not significant. 